\title{
Inclusive Breakup Theory of Three-Body Halos
}

\author{
Mahir S. Hussein ${ }^{1,2,3, \star}$, Lucas A. Souza ${ }^{1, \star \star}$, Emanuel Chimanski ${ }^{1, \star \star \star}$, Brett Carlson ${ }^{1, \star \star \star \star}$, and Tobias Frederico ${ }^{1, \dagger}$ \\ ${ }^{1}$ Instituto Tecnológico de Aeronáutica, DCTA, 12.228-900 São José dos Campos, SP, Brazil \\ ${ }^{2}$ Instituto de Estudos Avançados, Universidade de São Paulo C. P. 72012, 05508-970 São Paulo-SP, Brazil \\ ${ }^{3}$ Instituto de Física, Universidade de São Paulo, C. P. 66318, 05314-970 São Paulo,-SP, Brazil
}

\begin{abstract}
We present a recently developed theory for the inclusive breakup of three-fragment projectiles within a four-body spectator model [1], for the treatment of the elastic and inclusive non-elastic break up reactions involving weakly bound three-cluster nuclei in $A(a, b) X / a=x_{1}+x_{2}+b$ collisions. The four-body theory is an extension of the three-body approaches developed in the 80's by Ichimura, Autern and Vincent (IAV) [2], Udagawa and Tamura (UT) [3] and Hussein and McVoy (HM) [4]. We expect that experimentalists shall be encouraged to search for more information about the $x_{1}+x_{2}$ system in the elastic breakup cross section and that also further developments and extensions of the surrogate method will be pursued, based on the inclusive non-elastic breakup part of the $b$ spectrum.
\end{abstract}

\section{Introduction}

We report a recently developed theory to treat the inclusive breakup of three-fragment weakly bound nuclei [1]. The natural three fragment candidates for projectiles are Borromean, two-nucleon and unstable three-fragment halo nuclei. Our theory is an extension of the inclusive breakup models used for incomplete fusion reactions and in the surrogate method with two fragment projectiles. The threebody approach was developed in the 80's by Ichimura, Autern and Vincent (IAV) [2], Udagawa and Tamura (UT) [3] and Hussein and McVoy (HM) [4]. These three-body theories were extended to obtain the fragment yield in the reaction $A(a, b) X$, where the projectile is $a=x_{1}+x_{2}+b$. The inclusive breakup cross section is a sum of the fourbody elastic breakup cross section plus the inclusive nonelastic breakup cross section that involves the absorption cross sections of the participant fragments, $x_{1}$ and $x_{2}$, and which generalizes the three-body formula reviewed in Austern, et al. [5]. The connection between IAV, UT and HM theories was shown in [6].

The new formula contains the four-body dynamics both in the elastic breakup cross section and in the inclusive non-elastic breakup ones. It can be applied to treat reactions with stable/unstable projectiles composed of three-fragments, like weakly bound Borromean and two-nucleon halo nuclei, where fingerprints of Efimov physics [7] and universality [8] could be revealed through the appearance of long-range correlations between the three-fragments. In addition one can seek the generaliza-

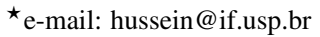

$\star \star$ e-mail: lucasufsj@gmail.com

$\star \star \star$ e-mail: evchimanski@gmail.com

$\star \star \star \star$ e-mail: brett@ita.br

†e-mail: tobias@ita.br
}

tion of the surrogate method applied to reactions like $(\mathrm{d}, \mathrm{p})$ and $(\mathrm{d}, \mathrm{n})$ to the reactions $(\mathrm{t}, \mathrm{d})$ and $\left({ }^{3} \mathrm{He}, \mathrm{d}\right)$, among others. Of particular interest is also the two-fragment correlation contribution to the elastic breakup cross section and the inclusive non-elastic breakup cross section through a threebody absorption interaction, which appears naturally in the four-body formulation. One could examine, for example, the relation between the pairing, in the case of two-neutron halos, and the three-body formulation of the projectile and its importance to the reaction mechanism underlying the inclusive breakup cross-sections.

We expect that these developments could stimulate experimentalists to search for more information about the $x_{1}+x_{2}$ system in the elastic breakup cross section, and that also further theoretical developments and extensions of the surrogate method will be pursued, based on the inclusive non-elastic breakup part of the $b$ spectrum.

\section{Inclusive Break-up theory}

The many-body Hamiltonian for the $b+x_{1}+x_{2}+A$ system to be applied to derive the scattering dynamics of the threefragment projectile is

$$
\begin{aligned}
H_{\left(b, x_{1}, x_{2}, A\right)}=T_{b}+ & T_{x_{1}}+T_{x_{2}}+V_{b, x_{1}}+V_{b, x_{2}}+V_{x_{1}, x_{2}} \\
& +h_{A}+T_{A}+V_{b, A}+V_{x_{1}, A}+V_{x_{2}, A}
\end{aligned}
$$

where the kinetic energies are given by the $T$ 's and the microscopic Hamiltonian of the target nucleus is $h_{A}$. In the spectator approximation the microscopic potential $V_{b, A}$ is associated by standard methods in reaction theory to the optical potential $U_{b}$, and the target is considered infinitely massive, namely $T_{A}=0$.

The inclusive breakup cross-section is an integral over the spectator fragment position $\mathbf{r}_{b}$ and a sum over the $x_{1}+$ 
$x_{2}+A$ bound and scattering states. This leads to the $b$ spectrum and angular distribution

$$
\begin{aligned}
\frac{d^{2} \sigma_{b}}{d E_{b} d \Omega_{b}} & =\frac{2 \pi}{\hbar v} \rho_{b}\left(E_{b}\right) \sum_{c} \delta\left(E-E_{b}-E_{c}\right) \\
& \times\left|\left\langle\chi_{b}^{(-)} \Psi_{x_{1} x_{2} A}^{c}\left|V_{b, x_{1}}+V_{b, x_{2}}+V_{x_{1}, x_{2}}\right| \Xi\right\rangle\right|^{2}
\end{aligned}
$$

where $\Xi\left(\mathbf{r}_{b}, \mathbf{r}_{x_{1}}, \mathbf{r}_{x_{2}}, A\right)$ is the exact eigenstate of the $A+$ $a$ many body Hamiltonian. The final state wave function is $\chi_{b}^{(-)}\left(\mathbf{k}_{b}, \mathbf{r}_{b}\right) \Psi_{\left(x_{1} x_{2} A\right)}^{c}$, where $\Psi_{\left(x_{1} x_{2} A\right)}^{c}$ runs over bound and continuum states. The density of $b$ continuum states is $\rho_{b}\left(E_{b}\right) \equiv\left[d \mathbf{k}_{b} /(2 \pi)^{3}\right] /\left[d E_{b} d \Omega_{b}\right]=\mu_{b} k_{b} /\left[(2 \pi)^{3} \hbar^{3}\right]$, with $\mu_{b}$ the reduced mass of the $b+A$ system.

The connection with the four-body (4B) scattering problem is obtained by eliminating the target internal degrees of freedom, and by using the product approximation, $\Xi=\Psi_{0}^{4 B(+)} \Phi_{A}$, where $\Psi_{0}^{4 B(+)}$ is the exact 4B scattering wave function in the incident channel, and $\Phi_{A}$ is the ground state wave function of the target nucleus. Together with the 4B approximation, the energy conservation $\delta$ in Eq. (2) is associated with the imaginary part of an optical model Green's function operator

$$
\operatorname{Im} G_{X}^{(+)}=-\pi \Omega_{X}^{(-)} \delta\left(E_{x}-H_{0}\right)\left(\Omega_{X}^{(-)}\right)^{\dagger}-\left(G_{X}^{(+)}\right)^{\dagger} W_{X} G_{X}^{(+)}
$$

where the Möller operator is $\Omega_{X}^{(-)}=\left[1+G_{X}^{(-)}\left(V_{X}\right)^{\dagger}\right]$. The imaginary part of the optical potential for particles $x_{1}$ and $x_{2}$ contains single fragment terms, $W_{x_{i}}$, and a three-body term. In addition, closure has to be used to perform the sum over $c$ and general nuclear reaction theory to transform the microscopic interactions $V_{x_{1}, A}$ and $V_{x_{2}, A}$ into complex optical potentials $U_{x_{1}}$ and $U_{x_{2}}$ (see e.g. [5]).

\subsection{Inclusive breakup cross-section}

Following the above steps, the inclusive breakup cross section is reduced to a sum of two distinct terms, the elastic breakup and the non-elastic breakup cross sections

$$
\frac{d^{2} \sigma_{b}}{d E_{b} d \Omega_{b}}=\frac{d^{2} \sigma_{b}^{E B}}{d E_{b} d \Omega_{b}}+\frac{d^{2} \sigma_{b}^{I N E B}}{d E_{b} d \Omega_{b}}
$$

with the $4 \mathrm{~B}$ elastic breakup cross section contribution being

$$
\begin{aligned}
\frac{d^{2} \sigma_{b}^{E B}}{d E_{b} d \Omega_{b}}= & \frac{2 \pi}{\hbar v_{a}} \rho_{b}\left(E_{b}\right) \int \frac{d k_{x_{1}}}{(2 \pi)^{3}} \frac{d k_{x_{2}}}{(2 \pi)^{3}} \\
& \times\left|\left\langle\chi_{x_{1}, x_{2}}^{3 B(-)} \chi_{b}^{(-)}\left|V_{b x_{1}}+V_{b x_{2}}\right| \Psi_{0}^{4 B(+)}\right\rangle\right|^{2} \\
& \times \delta\left(E-E_{b}-E_{\left(\mathbf{k}_{x_{1}}, \mathbf{k}_{x_{2}}\right)}\right)
\end{aligned}
$$

where $\chi_{x_{1}, x_{2}}^{3 B(-)}$ is the full scattering wave function of the two unobserved fragments in the final channel.

The inclusive cross-section for the inelastic breakup contains the optical potentials $U_{x_{1}}, U_{x_{2}}$ and the fragmentfragment interaction $V_{x_{1}, x_{2}}$ to all orders:

$$
\frac{d^{2} \sigma_{b}^{I N E B}}{d E_{b} d \Omega_{b}}=\frac{2}{\hbar v_{a}} \rho_{b}\left(E_{b}\right)\left\langle\hat{\rho}_{x_{1}, x_{2}}\left|W_{x_{1}}+W_{x_{2}}+W_{3 B}\right| \hat{\rho}_{x_{1}, x_{2}}\right\rangle
$$

where the source function

$$
\begin{aligned}
\hat{\rho}_{X}\left(\mathbf{r}_{x_{1}}, \mathbf{r}_{x_{2}}\right) & =\left(\chi_{b}^{(-)}\left|\Psi_{0}^{4 B(+)}\right\rangle=\right. \\
& =\int d \mathbf{r}_{b}\left[\chi_{b}^{(-)}\left(\mathbf{r}_{b}\right)\right]^{\dagger} \Psi_{0}^{4 B(+)}\left(\mathbf{r}_{b}, \mathbf{r}_{x_{1}}, \mathbf{r}_{x_{2}}\right)
\end{aligned}
$$

carries the full 4B dynamics in the optical model description. The imaginary parts of the optical potentials $U_{x_{1}}$ and $U_{x_{2}}$ are $W_{x_{1}}$ and $W_{x_{2}}$, respectively. Notice the presence of the imaginary part of a three-body optical potential $\left(W_{3 B}\right)$, associated with inelastic excitations that are irreducible to single fragment inelastic processes. This will be discussed in more detail in a later sections. We point out that the 4B inclusive non-elastic breakup cross section differs significantly from the 3B Austern formula [5].

The inelastic breakup cross-section is a sum of three terms,

$$
\frac{d^{2} \sigma_{b}^{I N E B}}{d E_{b} d \Omega_{b}}=\rho_{b}\left(E_{b}\right) \frac{k_{a}}{E_{a}}\left[\frac{E_{x_{1}}}{k_{x_{1}}} \sigma_{R}^{x_{1}}+\frac{E_{x_{2}}}{k_{x_{2}}} \sigma_{R}^{x_{2}}+\frac{E_{C M}\left(x_{1}, x_{2}\right)}{\left(k_{x_{1}}+k_{x_{2}}\right)} \sigma_{R}^{3 B}\right]
$$

where the approximation of a weakly bound projectile is used to write the kinetic energy: $E_{x_{i}, L a b}=E_{a, L a b}\left(M_{x_{i}} / M_{a}\right)$ with $M_{a}$ and $M_{x_{i}}$ being the mass numbers of the projectile and fragment, respectively. The single fragment inclusive cross-sections are

$\sigma_{R}^{x_{1}}=\frac{k_{x_{1}}}{E_{x_{1}}}\left\langle\hat{\rho}_{x_{1}, x_{2}}\left|W_{x_{1}}\right| \hat{\rho}_{x_{1}, x_{2}}\right\rangle, \quad \sigma_{R}^{x_{2}}=\frac{k_{x_{2}}}{E_{x_{2}}}\left\langle\hat{\rho}_{x_{1}, x_{2}}\left|W_{x_{2}}\right| \hat{\rho}_{x_{1}, x_{2}}\right\rangle$,

and the double fragment inclusive cross-section is

$$
\sigma_{R}^{3 B}=\frac{\left(k_{x_{1}}+k_{x_{2}}\right)}{E_{C M}\left(x_{1}, x_{2}\right)}\left\langle\hat{\rho}_{x_{1}, x_{2}}\left|W_{3 B}\right| \hat{\rho}_{x_{1}, x_{2}}\right\rangle
$$

which represents the two-fragment irreducible inelastic processes.

We elaborate here on the physical interpretation of the different contributions to the inclusive inelastic crosssection. The absorption of the fragment $x_{i}$ by the target is given by $\sigma_{R}^{x_{i}}(i=1,2)$, in the case in which the other fragment $x_{j}$ just scatters off the target through the optical potential $U_{x_{j} A}$. It is noteworthy to stress that $\sigma_{R}^{x_{i}}$ is different from the one in the $3 \mathrm{~B}$ theory of the $b-x-A$ system. The cross-sections $\sigma_{R}^{x_{1}}, \sigma_{R}^{x_{2}}$ are related to the three-body IAV cross section through a convolution of the latter with the distorted wave densities $\left|\chi_{x_{2}}^{(+)}\left(\mathbf{r}_{x_{2}}\right)\right|^{2}$, and $\left|\chi_{x_{1}}^{(+)}\left(\mathbf{r}_{x_{1}}\right)\right|^{2}$ of the spectator fragments $x_{1}$ and $x_{2}$, respectively. If an eikonal-type approximation of the projectile distorted wave $\chi_{a}^{(+)} \times \Phi_{a}\left(r_{b}, r_{x_{1}}, r_{x_{2}}\right)\left(a \equiv x_{1}+x_{2}+b\right)$ is used in the $4 \mathrm{~B}$ theory, the difference with the $3 \mathrm{~B}$ formulation is clearly exposed. Thus, it is expected that the $\sigma_{R}^{x_{1}}$ in a (t,p) reaction will differ from the $\sigma_{R}^{x}$ extracted in a $(\mathrm{d}, \mathrm{p})$ reaction. Furthermore, at low energies $\sigma_{R}^{x_{1}}, \sigma_{R}^{x_{2}}$, and $\sigma_{R}^{3 B}$ are associated with the formation of compound nuclei $A+x_{1}, A+x_{2}$ and $A+\left(x_{1}+x_{2}\right)$.

\subsection{Examples}

The first example to which we apply the 4B formulation of the inelastic breakup cross-sections is the case of the reaction ${ }^{9} \mathrm{Be}(=\alpha+\alpha+n)+{ }^{208} \mathrm{~Pb}$, where $4 \mathrm{~B}-\mathrm{CDCC}$ (fourbody Continuum Discretized Coupled Channels) calculations were performed by Descouvemont and collaborators 
[9] for the elastic scattering, elastic breakup, and total fusion cross-sections. However so far, CDCC cannot obtain the partial or incomplete fusion cross-sections. Despite this fact, we can point out that compound nuclei formation with $\alpha$ detected, namely $\alpha+{ }^{208} \mathrm{~Pb}={ }^{210} \mathrm{Po}, \mathrm{n}+{ }^{208} \mathrm{~Pb}=$ ${ }^{209} \mathrm{~Pb}$, and $\alpha+\mathrm{n}+{ }^{208} \mathrm{~Pb}={ }^{211} \mathrm{Po}$, would be very interesting to observe, in order to gather information about the spectrum of $\alpha$ particles in the analysis of the inclusive cross sections. It would be interesting to investigate the properties of these compound nuclei experimentally, as they are formed in such a hybrid reaction.

A second example is a two-neutron Borromean nuclei. The inclusive $\alpha$ spectrum for the ${ }^{6} \mathrm{He}$ projectile accounts for the formation of $\mathrm{n}+\mathrm{A}$ and $2 \mathrm{n}+\mathrm{A}$ compound nuclei. However it would be experimentally difficult to distinguish these in $\mathrm{CN}$ decay.

The third example is a two-proton Borromean nuclei. One might consider the inclusive proton spectra in the breakup of ${ }^{20} \mathrm{Mg}+{ }^{208} \mathrm{~Pb}$ with the formation of ${ }^{209} \mathrm{Bi},{ }^{226} \mathrm{U}$ and ${ }^{227} \mathrm{~Np}$ at different excitation energies. However, ${ }^{20} \mathrm{Mg}$ presents a very short lifetime and low intensity as a secondary beam.

\section{IB Cross Sections: HM Source function}

The source function computed with the Hussein and McVoy (HM) model [4] can be written as:

$$
\left\langle\mathbf{r}_{x_{1}}, \mathbf{r}_{x_{2}} \mid \hat{\rho}_{H M}^{4 B}\right\rangle=\hat{S}_{b}\left(\mathbf{r}_{x_{1}}, \mathbf{r}_{x_{2}}\right) \chi_{x_{1}}^{(+)}\left(\mathbf{r}_{x_{1}}\right) \chi_{x_{2}}^{(+)}\left(\mathbf{r}_{x_{2}}\right)
$$

where the four-body scattering state is approximated by the product of the distorted waves of the three fragments and the projectile bound state wave function. The internal motion modifies the $\mathrm{S}$-matrix of the $\mathrm{b}$ fragment as

$$
\hat{S}_{b}\left(\mathbf{r}_{x_{1}}, \mathbf{r}_{x_{2}}\right) \equiv \int d \mathbf{r}_{b} \Phi_{a}\left(\mathbf{r}_{x_{1}}, \mathbf{r}_{x_{2}}, \mathbf{r}_{b}\right)\left\langle\chi_{b}^{(-)} \mid \chi_{b}^{(+)}\right\rangle\left(\mathbf{r}_{b}\right),
$$

which should be compared to the 2B S-matrix element of $b$ given by $S_{\mathbf{k}_{b}^{\prime}, \mathbf{k}_{b}}=\int d \mathbf{r}_{b}\left\langle\chi_{b}^{(-)} \mid \chi_{b}^{(+)}\right\rangle\left(\mathbf{r}_{b}\right)$. In this approximation the inclusive cross-section in the 4B theory with $a=x_{1}+x_{2}+b$ is

$$
\begin{aligned}
\frac{E_{x_{1}}}{k_{x_{1}}} \sigma_{R}^{x_{1}}=\int d \mathbf{r}_{x_{1}} & \int d \mathbf{r}_{x_{2}}\left|\hat{S}_{b}\left(\mathbf{r}_{x_{1}}, \mathbf{r}_{x_{2}}\right)\right|^{2} \\
& \times\left|\chi_{x_{2}}^{(+)}\left(\mathbf{r}_{x_{2}}\right)\right|^{2} W\left(\mathbf{r}_{x_{1}}\right)\left|\chi_{x_{1}}^{(+)}\left(\mathbf{r}_{x_{1}}\right)\right|^{2},
\end{aligned}
$$

which should be contrasted with the cross-section in the 3B theory $(a=x+b)$

$$
\frac{E_{x}}{k_{x}} \sigma_{R}^{x}=\int d \mathbf{r}_{x}\left|\hat{S}_{b}\left(\mathbf{r}_{x}\right)\right|^{2} W\left(\mathbf{r}_{x}\right)\left|\chi_{x}^{(+)}\left(\mathbf{r}_{x}\right)\right|^{2}
$$

where $\hat{S}_{b}\left(\mathbf{r}_{x}\right) \equiv \int d \mathbf{r}_{b}\left\langle\chi_{b}^{(-)} \mid \chi_{b}^{(+)}\right\rangle\left(\mathbf{r}_{b}\right) \Phi_{a}\left(\mathbf{r}_{b}, \mathbf{r}_{x}\right)$. Comparing the cross-sections in Eqs. (12) and (13), one singles out the distorted wave $\left|\chi_{x_{2}}^{(+)}\left(\mathbf{r}_{x_{2}}\right)\right|^{2}$, which damps the 4B crosssection with respect to the $3 \mathrm{~B}$ one.

\section{$4 \mathrm{HM}$ and 3B Glauber theory}

We begin with the Glauber phase [10]

$$
\begin{array}{r}
{\left[\psi_{\vec{p}_{b}^{\prime}}^{(-)}\left(\vec{r}_{b}^{\prime}\right)\right]^{\star} \psi_{\vec{p}_{b}}^{(+)}\left(\vec{r}_{b}\right)=\exp \left[-i \frac{m}{\hbar p_{b}^{\prime}} \int_{-\infty}^{z_{b}^{\prime}} d z^{\prime} V\left(\sqrt{b_{b}^{\prime 2}+z^{\prime 2}}\right)\right.} \\
\left.-i \frac{m}{\hbar p_{b}} \int_{z_{b}}^{\infty} d z^{\prime} V\left(\sqrt{b_{b}^{2}+z^{\prime 2}}\right)-\imath \vec{q} \cdot \vec{r}_{b}\right],
\end{array}
$$

where $\hbar \vec{q}=\vec{p}_{b}^{\prime}-\vec{p}_{b}$, and $\vec{p}_{b}\left(\vec{p}_{b}^{\prime}\right)$ is the incoming (outgoing) momentum of the spectator. We take the initial value of the momentum to be $\vec{p}_{b}=p_{b} \hat{k}$ and the final value to be $\vec{p}_{b}^{\prime}=p_{b}^{\prime} \sin \theta \hat{i}+p_{b}^{\prime} \cos \theta \hat{k}$. The coordinates $\left(b_{b}^{\prime}, z_{b}^{\prime}\right)$ can be written in terms of the coordinates $\left(b_{b}, z_{b}\right)$ as

$$
\begin{aligned}
b_{b}^{\prime} & =b_{b} \cos \theta-z_{b} \sin \theta \\
z_{b}^{\prime} & =b_{b} \sin \theta+z_{b} \cos \theta .
\end{aligned}
$$

Figure 1 illustrates the coordinates $\left(b_{b}, z_{b}\right)$ and $\left(b_{b}^{\prime}, z_{b}^{\prime}\right)$.

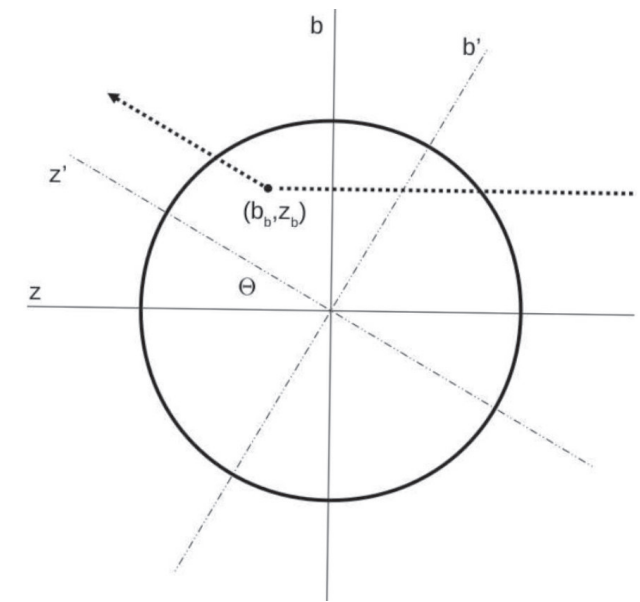

Figure 1. Appropriate kinematics and definitions of reference frame to compute the Glauber formula for the spectator distorted waves.

In order to get insight into the structure of the source function, we approximate the potential by a square well

$$
V(r)=\left(V_{0}-\imath W_{0}\right) \Theta(R-r) .
$$

The spectator distorted density then becomes

$$
\begin{gathered}
{\left[\psi_{\vec{p}_{b}^{\prime}}^{(-)}\left(\vec{r}_{b}^{\prime}\right)\right]^{\star} \psi_{\vec{p}_{b}}^{(+)}\left(\vec{r}_{b}\right)=\exp \left[-i \frac{m}{\hbar^{2}}\left(V_{0}+i W_{0}\right)\right.} \\
\left.\times\left(\frac{1}{p_{b}^{\prime}}\left(\sqrt{R^{2}-b_{b}^{\prime 2}}-z_{b}^{\prime}\right)+\frac{1}{p_{b}}\left(z_{b}+\sqrt{R^{2}-b_{b}^{2}}\right)\right)-\imath \vec{q} \cdot \vec{r}_{b}\right]
\end{gathered}
$$

for $b_{b}<R$ and $\exp \left[-l \vec{q} \cdot \vec{r}_{b}\right]$ for $b_{b} \geq R$.

The formula simplifies for the case of strong absorption, where the spectator distorted wave density becomes

$$
\left[\psi_{\vec{p}_{b}^{\prime}}^{(-)}\left({\overrightarrow{r^{\prime}}}_{b}\right)\right]^{\star} \psi_{\vec{p}_{b}}^{(+)}\left(\vec{r}_{b}\right)=\Theta\left(b_{b}-R\right) \Theta\left(b_{b}^{\prime}-R\right) \mathrm{e}^{-l \vec{q} \cdot \vec{r}_{b}} .
$$

Standard eikonal calculations ignore the difference $\left(b_{b}, z_{b}\right) \neq\left(b_{b}^{\prime}, z_{b}^{\prime}\right)$, inwhich case the distorted wave density can be simply written as

$$
\left[\psi_{\vec{p}_{b}^{\prime}}^{(-)}\left(\vec{r}_{b}^{\prime}\right)\right]^{\star} \psi_{\vec{p}_{b}}^{(+)}\left(\vec{r}_{b}\right)=\Theta\left(b_{b}-R\right) \mathrm{e}^{-l \vec{q} \cdot \vec{r}_{b}}
$$


The internal motion modified S-matrix of the $b$ fragment within the approximations above is given by

$$
\hat{S}_{b}\left(\mathbf{r}_{x_{1}}, \mathbf{r}_{x_{2}}\right)=\int d \mathbf{r}_{b} \Phi_{a}\left(\mathbf{r}_{x_{1}}, \mathbf{r}_{x_{2}}, \mathbf{r}_{b}\right) \Theta\left(b_{b}-R\right) \mathrm{e}^{-l \vec{q} \cdot \vec{r}_{b}}
$$

which produces a long range correlation between the fragments $x_{1}$ and $x_{2}$ for weakly bound projectiles, due to the long tail of the wave function penetrating in the classically forbidden region. This give us a taste of the possible röle of Efimov physics, for example when the projectile is a two-neutron halo s-wave state such as ${ }^{11} \mathrm{Li}$, in creating a long range correlation between the two neutrons. The actual set of coordinates to compute (21) can be visualized in Fig. 2.

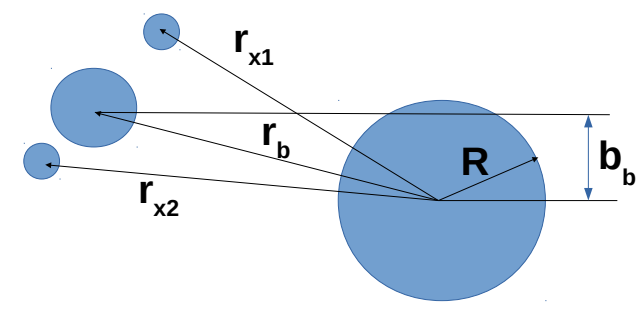

Figure 2. Definition of the coordinates of the fragments in configuration space. The target is represented by the largest circle, the unobserved fragments $x_{1}$ and $x_{2}$ are represented by the smallest circles, and the remaining circle represents the detected particle $b$.

Figure 2 also supplies a glimpse of the effect of an extended weakly bound system on the correlation between the two fragments $x_{1}$ and $x_{2}$. The formula (21) restricts $b$ to be outside the target absorptive region. However the tail of the projectile wave function can provide a long range correlation between the two fragments and the target.

It is instructive to calculate $\hat{S}_{b}\left(\mathbf{r}_{x_{1}}, \mathbf{r}_{x_{2}}\right)$ in the case of two-fragment projectiles, such as the deuteron in the $(d, p)$ reaction. Here $b=p$ and $x=n$, and we obtain, within the same strong absorption (black disk) eikonal approximation,

$$
\hat{S}_{p}\left(\mathbf{r}_{n}\right)=\int d \mathbf{r}_{p} \Phi_{d}\left(\mathbf{r}_{n}, \mathbf{r}_{p}\right) \Theta\left(b_{p}-R\right) \mathrm{e}^{-l \vec{q} \cdot \vec{r}_{p}}
$$

which corresponds to an incomplete Fourier transform involving $\mathbf{r}_{p}$ and $q$.

In the high energy regime, the cross section can be represented as an integral over impact parameter. Since Eq. (13),

$$
\frac{E_{x}}{k_{x}} \sigma_{R}^{x}=\int d \mathbf{r}_{x}\left|\hat{S}_{b}\left(\mathbf{r}_{x}\right)\right|^{2}\left[W\left(\mathbf{r}_{x}\right)\left|\chi_{x}^{(+)}\left(\mathbf{r}_{x}\right)\right|^{2}\right],
$$

contains the integrand of a b-integral of the reaction cross section of $x,\left[W\left(\mathbf{r}_{x}\right)\left|\chi_{x}^{(+)}\left(\mathbf{r}_{x}\right)\right|^{2}\right]$, which can be replaced by $\left[1-\left|S_{x}\left(b_{x}\right)\right|^{2}\right]$, and the factor $\left|\hat{S}_{b}\left(\mathbf{r}_{x}\right)\right|^{2}$ is basically the survival probability of the observed fragment, $b$, one can write, after rearranging terms,

$$
\sigma_{R}^{x}=2 \pi \int d b\left\langle\Phi_{a} \|\left. S_{b}(b)\right|^{2}\left[1-\left|S_{x}(b)\right|^{2}\right] \Phi_{a}\right\rangle
$$

One can say that the above equation is the high energy eikonal limit of IAV or the HM formulae. At high energy, the source function Eq.(6) for two-fragment projectiles reduces to $\hat{\rho}_{x}^{I A V}=\hat{\rho}_{x}^{H M}=\left(\chi_{b}^{(-)}\left|\chi_{b}^{(+)} \chi_{x}^{(+)} \Phi_{a}\right\rangle=\right.$ $\left\langle\chi_{b}^{(-)} \mid \chi_{b}^{(+)}\right\rangle\left|\chi_{x}^{(+)} \Phi_{a}\right\rangle$. When used in the expression for the cross section Eq. (13) or in its original form,

$$
\frac{d^{2} \sigma_{b}^{I N E B}}{d E_{b} d \Omega_{b}}=\frac{2}{\hbar v_{a}} \rho_{b}\left(E_{b}\right)\left\langle\hat{\rho}_{x}^{H M}\left|W_{x}\right| \hat{\rho}_{x}^{H M}\right\rangle,
$$

it is a simple exercise to reduce the expression to the Glauber cross section above. The above form of the cross section has been extensively used by $[11,12]$ to calculate one nucleon stripping and pickup reactions. These authors, take $\hat{R}_{1}=\left|\hat{S}_{b}\left(\mathbf{r}_{x}\right)\right|^{2}\left[W\left(\mathbf{r}_{x}\right)\left|\chi_{x}^{(+)}\left(\mathbf{r}_{x}\right)\right|^{2}\right]$ as an operator and calculate the expectation value in the state from which the nucleon is removed or added, $\left\langle\Phi_{i}\left|\hat{R}_{1}\right| \Phi_{i}\right\rangle$. This is then used to represent the cross section in a particularly transparent form. The partial cross section for removal of a nucleon, from a single-particle configuration $j^{\pi}$ populating the residue final state $\alpha$ with excitation energy $E_{\alpha}^{\star}$, is calculated as

$$
\sigma_{\alpha}=\left(\frac{A}{A-1}\right)^{N} C^{2} S\left(\alpha, j^{\pi}\right)\left\langle\Phi_{j^{\pi}}\left|\hat{R}_{1}\right| \Phi_{j^{\pi}}\right\rangle
$$

where $S_{\alpha}^{\star}=S_{n, p}+E_{\alpha}^{\star}$ is the effective separation energy for the final state $\alpha$ and $S_{n, p}$ is the ground-state to groundstate nucleon separation energy. Here the factor $N$, in the A-dependent center-of-mass correction factor that multiplies the shell-model spectroscopic factors $C^{2} S\left(\alpha, j^{\pi}\right)$, is the number of oscillator quanta associated with the major shell of the removed particle [11]. The cross section for the removal of a nucleon from the $j^{\pi}$ single particle shell is then the sum over all bound final states, $\alpha$ of the residual nucleus $A-1$.

Here, the radioactive projectile is now denoted by A. In this manner, useful nuclear structure information contained in the spectroscopic factor of the fragment in the projectile A, $C^{2} S\left(\alpha, j^{\pi}\right)$, can be extracted. Further, within the four-body theory, the practitioners of the eikonal theory extend their application to two nucleon removal or pickup. They use the following form of the reaction factor $\left\langle\Phi_{i}\left|\hat{R}_{2}\right| \Phi_{i}\right\rangle=\left\langle\left.\Phi_{i}|| S_{b}\right|^{2}\left|\left(1-\left|S_{x_{1}}\right|^{2}\right)\left(1-\left|S_{x_{2}}\right|^{2}\right)\right| \Phi_{i}\right\rangle$. This formula should come out from $\sigma_{R}^{3 B}$ of Eq. (9) of our theory in the high energy limit. However, there are several shortcomings in the above model, as there is no reference to the individual absorptions of $x_{1}$ and $x_{2}$. Further, the model above misses the correlation between the two interacting fragments. Currently we are investigating this point.

\section{Double fragment inelastic processes}

The inclusive cross-section expressed by $\sigma_{R}^{3 B}$ given by Eq. (9) is new and corresponds to genuine three-body absorption processes to inelastic channels. This part of the crosssection is associated with a three-body optical potential $U_{3 B}$ depending on the relative coordinates of the fragments $x_{1}, x_{2}$ and the target, and cannot be reduced to connected terms of the $3 \mathrm{~B}$ transition matrix with two-body potentials in different subsystems and the target in the ground state. 


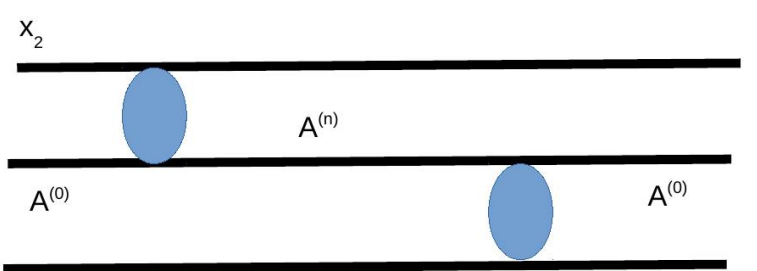

$x_{1}$

Figure 3. Three-body optical potential $U_{3 B}$. Excitation of the target by particle $x_{1}$ and de-excitation by $x_{2}$.

The structure of $W_{3 B}=\operatorname{Im}\left[U_{3 B}\right]$ results from conventional nuclear reaction theory. It includes, for example, processes like the virtual excitation of the target by one of the fragments and its virtual de-excitation by the other, as well as other $3 \mathrm{~B}$ processes irreducible to the re-scattering terms with the target in the ground state and with the final result being the full capture, or complete fusion, of both fragments. This is illustrated schematically in figure 3. If a resonant process exists, such as the excitation of giant pairing vibrations from the transfer of two neutrons from the projectile, it should furnish a large contribution to the optical three-body potential at the resonance energy.

\subsection{Hint on the 3B optical potential $U_{3 B}$}

The schematic figure 3 furnishes a path to formally build the the three-body optical potential by using projection operators out of the target ground state,

$$
\begin{aligned}
U_{3 B}=P V_{x_{1} A} Q & \left(Q G_{x A}\left(E_{x}\right) Q\right) Q V_{x_{2} A} P \\
& +P V_{x_{2} A} Q\left(Q G_{x A}\left(E_{x}\right) Q\right) Q V_{x_{1} A} P,
\end{aligned}
$$

where $E_{x}=E_{x_{1}}+E_{x_{2}}$ and the Q-projected 3B Green's function of the $x_{1}+x_{2}+A$ system is

$$
Q G_{x A} Q=\left[E_{x}-Q H_{0} Q+Q V_{x A} P G_{0} P V_{x A} Q+i \varepsilon\right]^{-1}
$$

with $V_{x A} \equiv V_{x_{1} A}+V_{x_{2} A}$. The imaginary part of the Qprojected Green's function of the $x_{1} x_{2} A \equiv x A$ system includes the virtual propagation in all states except for the elastic channel,

$$
\begin{aligned}
& \operatorname{Im}\left[Q G_{x A} Q\right]=-\pi \Omega_{Q}^{(-)} Q \delta\left(E_{x}-H_{0}\right) Q\left(\Omega_{Q}^{(-)}\right)^{\dagger} \\
& \quad+\left(Q G_{x A} Q\right)^{\dagger} Q V_{x A} P \delta\left(E_{x}-H_{0}\right) P V_{x A} Q G_{x A} Q
\end{aligned}
$$

Then, again using standard techniques, the imaginary part of $U_{3 B}$ can be isolated in (27) and can be written as

$$
\begin{aligned}
W_{3 B}= & U_{3 B}^{\dagger}-U_{3 B}= \\
= & \pi\left[P V_{x_{1} A} Q \Omega_{Q}^{(-)} Q \delta\left(E_{x}-H_{0}\right) Q\left(\Omega_{Q}^{(-)}\right)^{\dagger} Q V_{x_{2} A} P\right. \\
+ & P V_{x_{1} A} Q\left(Q G_{x A} Q\right)^{\dagger} Q V_{x A} P \delta\left(E_{x}-H_{0}\right) P \\
& \left.\times P V_{x A} Q\left(Q G_{x A} Q\right) Q V_{x A} P\right]+\left(x_{1} \leftrightarrow x_{2}\right)
\end{aligned}
$$

where one can identify the virtual propagation of the $x_{1} x_{2} A$ system through the inelastic channels in the Q-space.

\section{Beyond HM, UT, and IAV formulas}

The key points for going beyond the IAV formula for the inclusive inelastic cross-section are to include in the source term the full four-body dynamics and the threebody optical potential, by computing $\left|\Psi_{0}^{4 B(+)}\right\rangle$ without approximating the four-body problem. For the time being we will be content to generalize the three-body IAV formula to the four-body case and present the $\mathrm{CFH}$ formula given in [1].

The generalization given by $\mathrm{CFH}$ of the IAV formula to determine the inclusive cross-section observed by the detection of the spectator particle $b$, contains HM and UT like terms. The steps to derive a $4 \mathrm{~B}$ version of the IAV formula follow [6] and we write

$$
\left|\Psi_{0}^{4 B(+)}\right\rangle=G_{b, x_{1}, x_{2}, A}^{(+)} V_{x}\left|\Psi_{0}^{4 B(+)}\right\rangle \approx G_{b, x_{1}, x_{2}, A}^{(+)} V_{x}\left|\chi_{a}^{(+)}, \Phi_{a}\right\rangle
$$

where $V_{x}=V_{b x_{1}}+V_{b x_{2}}$ is the interaction between the detected fragment and the other two, $\left|\Phi_{a}\right\rangle$ the projectile internal wave function and $\chi_{a}^{(+)}$the distorted wave of the center of mass. The four body Green's function is

$G_{b, x_{1}, x_{2}, A}^{(+)}=\left[E-T_{b}-T_{x_{1}}-T_{x_{2}}-V_{x_{1}, x_{2}}-U_{b}-U_{x_{1}}-U_{x_{2}}+i \varepsilon\right]^{-1}$.

where we have not explicitly included $U_{3 B}$, which should also contribute to the Green's function.

The generalization of the IAV three-body source function for the four-body case is:

$$
\hat{\rho}_{x_{1}, x_{2}}^{C F H}=\left(\chi_{b}^{(-)}\left|\Psi_{0}^{4 B(+)}\right\rangle=\left\langle\chi_{b}^{(-)}\left|G_{b, x_{1}, x_{2}, A}^{(+)} V_{x}\right| \chi_{a}^{(+)} \Phi_{a}\right\rangle\right.
$$

By manipulating the above formula in analogy to the development provided in Ref. [6], one gets the 4B extension of the 3B Ichimura-Austern-Vincent as

$$
\hat{\rho}_{x_{1}, x_{2}}^{C F H}=\hat{\rho}_{x_{1}, x_{2}}^{U T}+\hat{\rho}_{x_{1}, x_{2}}^{H M}
$$

where the 4B Hussein-McVoy (HM) term is

$$
\hat{\rho}_{x_{1}, x_{2}}^{H M}=\left\langle\chi_{b}^{(-)} \mid \chi_{a}^{(+)} \Phi_{a}\right\rangle
$$

and the 4B Udagawa-Tamura (UT) term is

$$
\hat{\rho}_{x_{1}, x_{2}}^{U T} \equiv G_{x_{1}, x_{2}, A}^{(+)}\left\langle\chi_{b}^{(-)}\left|\left[U_{b}+U_{x_{1}}+U_{x_{2}}-U_{a}\right]\right| \chi_{a}^{(+)} \Phi_{a}\right\rangle .
$$

where $U_{a}$ is the optical potential of the projectile which generates its distorted wave, $\chi_{a}^{(+)}$.

The Green's function

$$
G_{x_{1}, x_{2}, A}^{(+)}=\left[E-E_{b}-T_{x_{1}}-T_{x_{2}}-V_{x_{1}, x_{2}}-U_{x_{1}}-U_{x_{2}}+i \varepsilon\right]^{-1}
$$

should also contain in principle the three-body optical potential. Further studies of this particular point will be developed.

\section{Inclusive breakup and Efimov Physics}

The inclusive cross-section formulae for the elastic breakup (4) and for the inelastic process (5) applied to a three-body halo nuclei, e.g., a weakly bound neutronneutron-core nuclei close to the drip-line, contains a longrange correlation between the two neutrons and the core 
$b$, through the extended wave function of the halo and the scattering wave $\chi_{x_{1}}^{(+)}\left(\mathbf{r}_{x_{1}}\right) \chi_{x_{2}}^{(+)}\left(\mathbf{r}_{x_{2}}\right) \chi_{x_{b}}^{(+)}\left(\mathbf{r}_{x_{b}}\right)$, which appears both in (4) and in the source function in (5), when such an approximation is made.

However, we expect that interesting physics remains beyond such an approximation if the 4B scattering wave function, $\Psi_{0}^{4 B(+)}\left(\mathbf{r}_{b}, \mathbf{r}_{x_{1}}, \mathbf{r}_{x_{2}}\right)$ is taken in full. It should contain dynamics of the continuum of the neutron-neutroncore system beyond the bound halo state. The reaction mechanism now includes Efimov physics [7] and with that the long range correlation between the halo fragments, which could be influenced by the presence of Efimov bound, virtual or resonant states [8] (see also [13] for a discussion of observing Efimov states in halo reactions). Indeed, the existence of Borromean Efimov states was observed about a decade ago [14] in the resonant three-body recombination of cold cesium atoms in magneto-optical traps.

\section{Digression on two-fragment projectile inclusive breakup cross section}

It is important to remind the reader of the advances made in the application of the inclusive breakup theory of twofragment projectiles, as was originally developed in [25]. Quite recently this theory was applied to the $(\mathrm{d}, \mathrm{p})$ reaction [19-21, 23] as a mean to test the validity of the Surrogate Method [24-26], employed to extract neutron capture and fission cross sections of actinide target nuclei, such as ${ }^{238} \mathrm{U},{ }^{232} \mathrm{Th}$, of importance for the development of next generation fast breeder reactors. These neutron capture reactions on other targets are also important for the study of element formation following supernova explosion through the astrophysical s-process. The general conclusion of [19-21, 23] was that the Surrogate Method, the extraction of the cross section for the formation and decay of the compound nucleus of the $(n+A)$ subsystem, as well as the total capture cross section (the cross section for the formation of the compound nucleus), is justified as long as the direct part of the cross section is calculated and subtracted from the inclusive breakup total reaction cross section $\sigma_{R}^{(n+A)}$. Another topic of importance is the Trojan Horse Method [27-30] used to extract from the inclusive breakup data a direct reaction of interest to nuclear astrophysics following nova explosion, which otherwise would be difficult to measure in the laboratory. The THM uses the advantage that the surrogate charged fragment $x$ is a bare nucleus with no electrons surrounding it. This avoids the problem of electron screening. Further, since this fragment is brought by the primary projectile to the region of the target, the Coulomb barrier is already surmounted, and accordingly no hindrance due to barrier penetration and tunneling is present in the $x$ induced reaction. The two methods, SM, and THM, are two pieces of the same quantity, namely $\sigma_{R}^{(x+A)}$ in the breakup reaction, $a+A \rightarrow b+(x+A)$. Thus the THM can be easily justified within the theory alluded to above as being a process contributing to the direct part of $\sigma_{R}^{(x+A)}$. As a final remark, if the surrogate fragment $x$ is a neutron then the SM and the THM are the same!
It would certainly be important to extend these studies to the case of the three-fragment projectiles discussed in this contribution. In particular, it would be particularly interesting to extend the THM to cases involving threefragment projectiles, where three types of reactions can be extracted, $x_{1}+A \rightarrow y_{1}+B_{1}, x_{2}+A \rightarrow y_{2}+B_{2}$, and $x_{1}+x_{2}+A \rightarrow y_{3}+B_{3}$. Work along these lines is in progress.

\section{Conclusions and Perspectives}

We have reported in this contribution that the general structure of the CFH cross section in the DWBA limit is similar in structure to the $3 \mathrm{~B}$ one, with the full post form (or four-body IAV), which can be written as the sum of the prior four-body UT cross section plus the four-body HM one plus an interference term when the 4B source term detailed in Eq. (33) is used to compute the inelastic part of the inclusive cross-section. The major difference between the $4 \mathrm{~B}$ and $3 \mathrm{~B}$ cases resides in the structure of the reaction cross sections for the absorption of one of the interacting fragments, which we find to be damped by the absorption effect of the other fragment. It is expected that in a (t,p) reaction, an absorption cross section of the $n+A$ subsystem would be smaller than the corresponding one in a (d,p) reaction. Another important new feature is the $3 \mathrm{~B}$ absorption from a three-body optical potential, the formal structure of which we have sketched.

The perspectives of our study are in the use of the Faddeev-Yakubovski equations $[15,16]$ to develop an expansion of the four-body wave function, to attempt to go beyond the CFH/IAV formulas. This effort could also be accomplished by building the four-body CDCC wave function to compute the source term. In addition, we can ask about the dynamics that are built in the three-body optical potential when giant pairing vibrations (GPV) are possible $[17,18]$. These collective states involve the coherent excitation of particle-particle pairs, in complete analogy to the coherent excitation of particle-hole pairs that constitutes the microscopic foundation of multipole giant resonances. For example, the GPV opens interesting prospects for nuclear structure studies in reactions of the type $(\mathrm{t}, \mathrm{p}), 2 \mathrm{n}$ Borromean cases such as $\left({ }^{6} \mathrm{He},{ }^{4} \mathrm{He}\right),\left({ }^{11} \mathrm{Li}\right.$, $\left.{ }^{9} \mathrm{Li}\right),\left({ }^{14} \mathrm{Be},{ }^{12} \mathrm{Be}\right),\left({ }^{22} \mathrm{C},{ }^{20} \mathrm{C}\right)$, and the $2 \mathrm{p}$ halo cases, $\left({ }^{17} \mathrm{Ne}\right.$, $\left.{ }^{15} \mathrm{O}\right)$, and $\left({ }^{20} \mathrm{Mg},{ }^{18} \mathrm{Ne}\right)$. The theory we have developed is an appropriate framework to study these types of collective nuclear excitations, associated with pairing correlations in the target.

From the point of view of a two-neutron s-wave dominated halo target our theory naturally leads to an inquiry about the röle of Efimov physics in inclusive breakup cross-section, both in the elastic and inelastic contributions, which should have its place beyond the $\mathrm{CFH}$ formula.

Further, the theory can be extended to allow for inclusive reactions where two or more fragments are detected. We expect that the developments discussed in our recent work should stimulate experimental and theoretical works to seek more information about the $x_{1}+x_{2}$ system in the elastic and inelastic breakup cross sections, and in particular to theorists to extend the 
Surrogate Method and the Trojan Horse Method, both based on the inclusive non-elastic breakup part of the $b$ spectrum in a two-fragment projectile induced reaction, to three-fragment projectiles.

Acknowledgements. This work was partly supported by the Brazilian agencies, Fundação de Amparo à Pesquisa do Estado de São Paulo (FAPESP), the Conselho Nacional de Desenvolvimento Científico e Tecnológico (CNPq). MSH also acknowledges a Senior Visiting Professorship granted by the Coordenação de Aperfeiçoamento de Pessoal de Nível Superior (CAPES), through the CAPES/ITAPVS program.

\section{References}

[1] B. V. Carlson, T. Frederico, M. S. Hussein, Phys. Lett. B 767, 53 (2017).

[2] M. Ichimura, N. Austern, and C. M. Vincent, Phys. Rev. C 32, 431 (1985).

[3] T. Udagawa and T. Tamura, Phys. Rev. C 24, 1348 (1981).

[4] M. S. Hussein and K. W. McVoy, Nucl. Phys. A 445, 124 (1985)

[5] N. Austern, Y. Iseri, M. Kamimura, M. Kawai, G. Rawitscher, and M. Yahiro, Phys. Rep. 154, 125 (1987).

[6] M. S. Hussein, T. Frederico, and R. C. Mastroleo, Nucl. Phys. A 511, 269 (1990).

[7] V. Efimov, Phys. Lett. B 33, 563 (1970) 563; FewBody Syst. 51, 79 (2011).

[8] T. Frederico, M.T. Yamashita, A. Delfino, L. Tomio, Prog. Part. Nucl. Phys. 67, 939 (2012).

[9] P. Descouvemont, T. Druet, L. F. Canto, and M. S. Hussein Phys. Rev. C 91, 024606 (2015).

[10] L. F. Canto and M. S. Hussein, Scattering Theory of Molecules, Atoms and Nuclei, World Scientific , 2013

[11] P. G. Hansen and J. A. Tostevin, Annu. Rev. Nucl. Part. Sci. 53, 219 (2003).

[12] H. Esbensen and G. F. Bertsch Phys. Rev. C 64, 014608 ( 2001)
[13] A. O. Macchiavelli, Few-Body Syst. 56, 773 (2015)

[14] T. Kraemer, et al., Nature 440, 315 (2006).

[15] L.D.Faddeev, Zh. Eksp. Teor. Fiz. 39, 1459 (1960) [Sov. Phys. JETP 12, 1014 (1961)].

[16] O. A. Yakubovsky, Yad. Fiz. 5, 1312 (1967) [Sov. J. Nucl. Phys. 5, 1312 (1967)].

[17] R. A. Broglia and D. R. Bes, Phys. Lett. B 69, 129 (1977).

[18] F. Cappuzzello, D. Carbone, M. Cavallaro, M. Bond, C. Agodi, F. Azaiez, A. Bonaccorso, A. Cunsolo, L. Fortunato, A. Foti, S. Franchoo, E. Khan, R. Linares, J. Lubian, J. A. Scarpaci and A. Vitturi, Nature Communications 6, 6743 (2015).

[19] Q. Ducasse et al., arXiv:1512.06334 [nucl-ex]

[20] G. Potel, F. M. Nunes, and I. J. Thompson, Phys. Rev. C 92, 034611 (2015).

[21] J. Lei and A. M. Moro, Phys. Rev. C 92, 044616 (2015); J. Lei and A. M. Moro, C 92, 061602(R) (2015).

[22] G. Potel et al. To be published in the European Physical Journal A (2017)

[23] B.V. Carlson, R. Capote, M. Sin, Few-Body Syst. 57, 307 (2016).

[24] Jutta E. Escher and Frank S. Dietrich, Phys. Rev. C 74, 054601 (2006)

[25] Jutta E. Escher, Jason T. Burke, Frank S. Dietrich, Nicholas D. Scielzo, Ian J. Thompson, and Walid Younes, Rev. Mod. Phys., 84, 353 (2012)

[26] J. E. Escher, A. P. Tonchev, J. T. Burke, P. Bedrossian, R. J. Casperson, N. Cooper, R. O. Hughes, P. Humby, R. S. Ilieva,, S. Ota1, N. Pietralla,, N. D. Scielzo, and V.Werner, EPJ Web of Conferences 122, 12001 (2016)

[27] S. Typel and G. Baur, Ann. Phys. (NY) 305, 228 (2003)

[28] C. Spitaleri et al., Phys. of Atomic Nuclei 74, 1725 (2011)

[29] A. Tumino et al., Few Body Systems, (2012) DOI 10.1007/s00601-012-0407-1

[30] R. Tribble et al., Rep. Prog. Pays. 77, 106901(2014) 